over-expression of PGC-1 $\beta$ promoted migration and invasion of RA-FLS (all $p<0.05$, figure $1 A \sim C)$. Down-regulation of $P G C-1 \beta$ in RA-FLS significantly decreased the expression of MMP-3 and MMP-9 in the culture supernatant which was measured by Proteome Profiler human protease array (MMP-3: $p=0.032$, MMP-9: $p=0.037)$. Further $q R T-P C R$ and western blot analysis verified that both mRNA and protein expression of MMP-3 and MMP-9 in RA-FLS were significantly decreased compared with Lv-sh-GFP transfection group (all $p<0.05$, figure 1D). Down-regulated PGC-1 $\beta$ in RA-FLS significantly suppressed the expression of

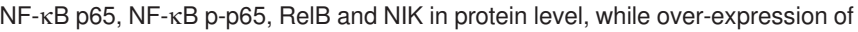
PGC-1 $\beta$ promoted the expression of NF- $\kappa B$ p65, NF- $\kappa B$ p-p65, RelB and NIK (all $\mathrm{p}<0.05$, figure $1 \mathrm{E})$.
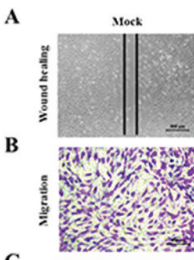

C
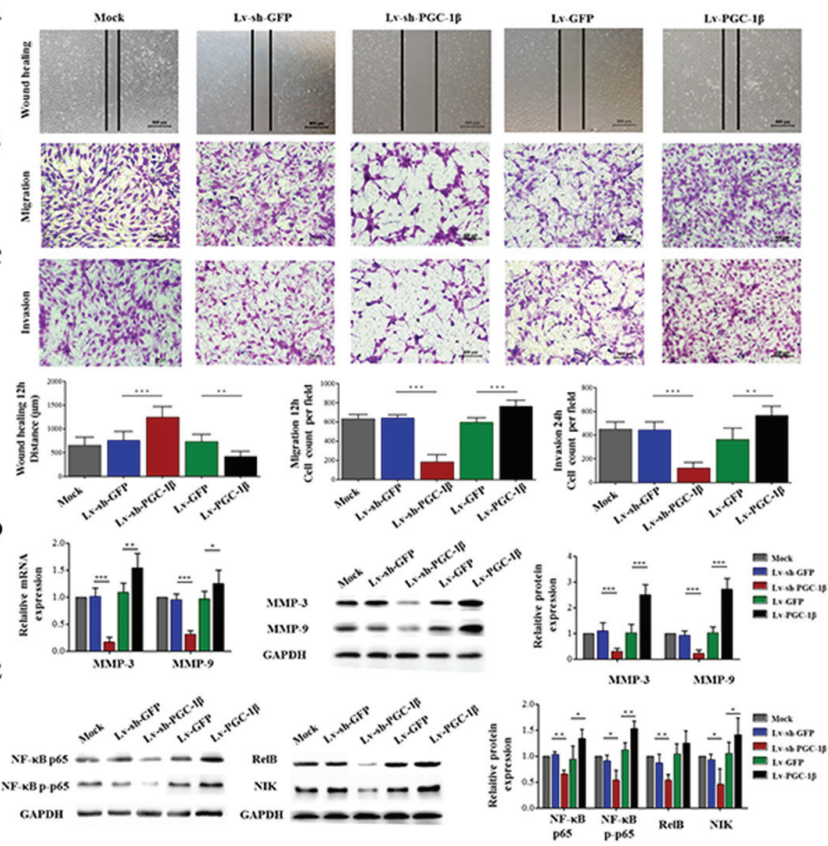

Abstract AB0098 - Figure 1. Effects of PGC-1 $\beta$ on the migration and invasion capacity as well as proteases expression, canonical and non-canonical NF-KB signalling pathway activation in RA-FLS. A C After down-regulation or over-expression of PGC-1 $\beta$, wound healing and transwell assay showed the capacity of horizontal migration $(\mathbf{A})$, vertical migration (B) and invasion (C) of primary RA-FLS. D After down-regulation or overexpression of PGC-1 $\beta$, the expression of MMP-3 and MMP-9 was measured by qRTPCR and western blot. E After down-regulation or overexpression of PGC-1 $\beta$, the

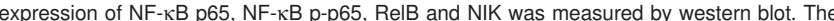
data were representative as means \pm SD from six different RA patients. ${ }^{*} P<0.05$, ${ }^{\star *} P<0.01$, ${ }^{\star \star \star} P<0.001$.

Conclusions: Our findings suggested that PGC-1 $\beta$ facilitates the migration and invasion capacity and MMP-3/9 expression in RA-FLS through activation canonical and non-canonical NF- $\mathrm{KB}$ signalling pathway.

Acknowledgements: This work was supported by National Natural Science Foundation of China (no. 81471597 and 81671612), Guangdong Natural Science Foundation (no. 2016A030313307, 2017A030313576 and 2017A030310236) and Guangdong Medical Scientific Research Foundation (no. A2017109).

Disclosure of Interest: None declared

DOI: 10.1136/annrheumdis-2018-eular.5103

\section{AB0099 ARTHROSCOPIC PECULIARITIES OF THE INFLAMMATORY PROCESS OF SYNOVIAL SHELL IN UROGENIC ARTHRITIS}

M. Salokhiddinov, I. Xodjanazarov. Traumatology, Tashkent Medical Academy, Tashkent, Uzbekistan

Background: In recent years there has been an increase in the incidence of reac tive arthritis $(\operatorname{Re} A)$, associated with urogenital infection. Chronic synovitis of urogenital etiology is a nonspecific process, pathogenesis is very close to rheumatoid arthritis (RA), which complicates differential diagnosis. According to the literature, the inflammatory process in the synovial membrane (SM) supports the persistence of chlamydia, which reduces the effectiveness of standard anti- inflammatory therapy of arthritis in patients with undifferentiated seronegative oligoarthritis, spondyloarthropathy, RA.

Objectives: Arthroscopic assessment of the level of the inflammatory process in the synovial membrane, depending on the duration of the disease and the activity of the pathological process in patients with urogenital arthritis.

Methods: The study involved 39 patients with RA of urogenital etiology complicated by synovitis of knee joint (KJ) were examined; 23 of them were women and 16 men; mean age was $35.5 \pm 38$.0 years. They received inpatient treatment at the Department of Traumatology and Orthopaedics of the 2nd TMA Clinic in 20142016 years. All these patients underwent diagnostic arthroscopy of KJ. Laboratory tests included enzyme-linked immunosorbent assay (ELISA) for TORCH infection, a polymerase reaction (PCR), determination of a rheumatic factor in synovia fluid and blood before and after treatment, arthroscopy of the joint.

Results: In arthroscopic examination in the acute phase (early period) of ReA dull SM, vasodilation, and oedema are revealed, which is accompanied by an increase in the production of turbid synovial fluid (SF). On the surface of SM there are sections of fibrin filaments, expansion and hyperemia along the vessels. In the long-term (subacute phase) these violations partially disappear. The surface of the synovium can again become single-rowed, with moderate hyperemia of the vessels. The deposits of fibrin on the entire surface of SM can be significant. However, with reduced immunoreactivity of the organism, the reactive synovitis persists for a long time, and the chronic course of the pathological process leads to abundant deposits of fibrin masses and to the formation of villi in the form of large and flat petals. In some parts of the vascular vessels are pale, accompanied by disorganisation of the collagen frame of SM, which directly affects the cells of the articular cartilage.

Conclusions: Thus, reactive urogenital arthritis due to the persistence of an infectious agent is characterised by the polymorphism of the damage to the joint and cartilage of the joint. The arthroscopic changes revealed at various stages of the inflammatory process reflect the reactivity of the pathological process and determine the extent of the lesion. Arthroscopic examination of biopsy specimens of SM and cartilage allows to determine the dynamics of the disease, the degree of lesion, evaluate the effectiveness of the preventive and therapeutic measures being carried out, and also to determine the indications for joint synovcapsullectomy.

\section{REFERENCES}

[1] Sweeney SE, et al. Clinical features of rheumatoid arthritis. In GS Firestein, et al., (eds.), Kelley's Textbook of Rheumatology, 9th ed., 2013;2:1109-1136. Philadelphia: Saunders.

[2] Yasuda GT, et al. Rheumatoid arthritis. In JE Pizzorno, MT Murray, (eds.), Textbook of Natural Medicine, 4th ed., 2013:1769-1784. St. Louis: Elsevier.

Disclosure of Interest: None declared

DOI: 10.1136/annrheumdis-2018-eular.7027

\section{AB0100 \\ NEUROGENIC INFLAMMATION CHARACTERISED BY NERVE GROWTH FACTOR, TRKA AND SUBSTANCE P IS PREVALENT IN HUMAN FACET JOINT OSTEOARTHRITIS}

M.F. Seidel ${ }^{1}$, J. Geurts ${ }^{2}$, N. Busso ${ }^{3}$, V. Chobaz ${ }^{3}$, T. Hügle $^{3} .{ }^{1}$ Rheumatology, Schmerzklinik Basel; ${ }^{2}$ Spine Surgery, University Hospital of Basel, Basel; ${ }^{3}$ Rheumatology, University Hospital Lausanne (CHUV), Lausanne, Switzerland

Background: Facet joint osteoarthritis (FOA) is a frequent cause of chronic low back pain and spinal stenosis. Neuron-derived molecules regulate pain sensation and inflammation. Nerve growth factor (NGF) is one of the most important mediators for this mechanism that is termed neurogenic inflammation. ${ }^{1}$ Among many other effects, it regulates substance $P(S P)$ expression as pivotal downstream peripheral pro-inflammatory molecule. Consequently, NGF inhibitors (NGFi) as a novel class of pain medication have shown significant efficacy in $O A$ and to some extent also low back pain. However, it is unknown which tissue compartments in facet joints are involved in NGF signalling.

Objectives: To determine expression patterns of NGF, its high affinity transmembrane tyrosine kinase $\mathrm{A}$ (TrkA) receptor and SP in cartilage, subchondral bone marrow and capsular tissues of facet joints (FJ).

Methods: Dissected human FOA specimens of six donors were examined. OA severity was graded on HE-stained tissue sections. NGF, TrkA and SP expres sion was evaluated by immunohistochemistry with monoclonal antibodies. Similarly, new bone formation was assessed by staining for osteocalcin.

Results: FJ had low $(n=2)$, high $(n=2)$ and intermediate $(n=2)$ inflammatory bone marrow infiltrates. NGF was strongly expressed in capsular tissue (figure, 40x), 